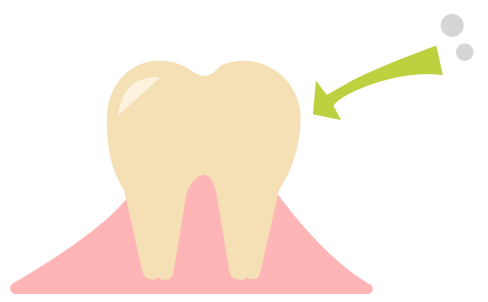

\title{
ANÁLISE DA UTILIZAÇÃO DE NANOPARTÍCULAS DE PRATA E/OU PLASMA DE BAIXA PRESSÃO E TEMPERATURA PARA A PREVENÇÃO DE CANDIDA ALBICANS EM PRÓTESES DENTÁRIAS
}

\author{
EVALUATION OF PREVENTION OF CANDIDA ALBICANS IN DENTAL \\ PROSTHESES AFTER USING OF SILVER NANOPARTICLES AND PRESSURE \\ PLASMA PERNAMBUCO: DATASUS DATA-BASED MAPPING
}

\begin{abstract}
Edeviane Stieven
Cirurgiã-dentista. Graduada em Odontologia pelo Centro Universitário da Serra Gaúcha, Caxias do Sul/RS. E-mail: divi_stieven@hotmail.com
\end{abstract}

\section{Alexandre Conde}

Professor do Curso de Graduação em Odontologia do Centro Universitário da Serra Gaúcha. Doutor em Materiais Dentários pela PUC/RS (2007). E-mail: alexandre.conde@fsg.br

\section{Daniel Galafassi}

Professor do Curso de Graduação em Odontologia do Centro Universitário da Serra Gaúcha. Doutor em Odontologia Restauradora pela USP (2012). Mestre em Dentística pela USP (2009). E-mail: daniel.galafassi@fsg.br

\section{Estelamari Barbieri Elsemann}

Professor do Curso de Graduação em Odontologia do Centro Universitário da Serra Gaúcha. Mestre em Radiologia Odontológica e Imaginologia pelo Centro de Pesquisas Odontológicas São Leopoldo Mandic (2016). E-mail: estela.elsemann@fsg.br

\section{Rogério Brasiliense Elsemann}

Professor do Curso de Graduação em Odontologia do Centro Universitário da Serra Gaúcha. Doutor em Odontologia pela PUC/RS (2007). Mestre em Odontologia pelo Centro de Pesquisas Odontológicas São Leopoldo Mandic (2003). E-mail: rogerio.elsemann@fsg.br

\section{Alexandra Flavia Gazzoni}

Professor do Curso de Graduação em Odontologia do Centro Universitário da Serra Gaúcha. PhD em Microbiologia pela Universitat Rovira i Virgili (Catalunya-Espanha) (2010). Doutora em Ciências Pneumológicas pela UFRGS (2009). Mestre em Medicina (Pneumologia) pela UFRGS (2007). Responsávelchefe do Laboratório de Microbiologia e Patologia Bucaldo Centro Universitário da Serra Gaúcha. E-mail: alexandra.gazzoni@fsg.br

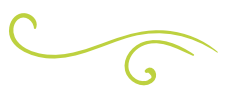

\section{RESUMO}

Objetiva-se investigar a contribuição da adição de nanopartículas de prata e/ou plasma a baixa pressão e temperatura na adesão da Candida albicans em superfícies de base

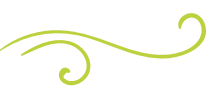

acrílica. Para tanto, foram confeccionadas 80 amostras padrão de poli(metacrilato de metila) com dimensões de $2 \mathrm{~cm} \times 1 \mathrm{~cm} \times$ $3 \mathrm{~mm}$, sendo divididos em 4 grupos: (G1) grupo controle; (G2) adição de 0,3\% de 
nanopartículas de prata, somente; (G3) adição de banho de plasma de baixa pressão e temperatura na superfície da resina acrílica; (G4) adição de 0,3\% de nanopartículas de prata acrescidas debanho de plasma à baixa pressão e temperatura. Todos os corpos de prova foram contaminados com C. albicans a partir de uma solução contendo 107 células/mililitros. Após 24 horas da contaminação, os corpos de prova foram removidos e, subsequentemente, houve a determinação da adesão fúngica através da contagem das unidades formadoras de colônias por $\mathrm{mL}$. Os dados foram analisados utilizando o teste de ANOVA-one way, seguido pela análise de comparações múltiplas pelo teste de Tukey's. Quando o grupo-controle foi comparado aos grupos 2, 3 e 4, observou-se diferença significativa entre eles $(P<0,0001)$. Quando comparados os grupos testes, não se observou diferença estatisticamente significativa. A adição de nanopartículas de prata de forma individual, bem como o uso de plasma a baixa pressão e temperatura utilizado de forma isolada ou em conjunto a adição de nanopartículas de prata, parece ser uma estratégia promissora na prevenção de estomatite protética, diminuindo a adesão fúngica em resinas acrílicas.

PALAVRAS-CHAVE: Candidíase Bucal. Estomatite. Nanotecnologia.

\section{ABSTRACT}

This study evaluated the effect of incorporation of a silver nanoparticule solution or/ and non-thermal atmospheric-pressure cold plasma jet in a denture base acrylic resin against Candida albicans adhesion. Therefore, specimens $(n=80)$ of methyl polymethyl methacrylate measuring $2 \mathrm{~cm} \times 1 \mathrm{~cm}$ $\times 3 \mathrm{~mm}$ were randomly fabricated and were divided in 4 groups: (G1) control group, (G2) incorporation of $0.3 \mathrm{wt} \%$ of silver nanoparticles only; (G3) Atmospheric-pressure cold plasma applied to process the surface of heat-polymerized acrylic resin only; (G4) with both associated technologies (incorporation of $0.3 w t \%$ of silver nanoparticles into a denture base acrylic resin plus Atmosphericpressure cold plasma applied to process the surface of heat-polymerized acrylic resin). The AgNPs solution was synthesized by chemical reduction. In antimicrobial assay, specimens were contaminated with a Candida albicans suspension spectrophotometrically standardized at of $1 \times 107 \mathrm{CFU} / \mathrm{mL}$. and then incubated at $37 \circ C$ for 24 hours. After this period, the amount of Candida albicans cells adhering to acrylic specimen surfaces was evaluated by colony- forming unit (CFU) per ml. assay. The data were analyzed by one-way ANOVA followed by Tukey's HSD post hoc test $(p<0.05$ ). Significant reduced CFU was exhibited when the control group was compared to the groups 2, 3 and 4 ( $P<0.0001)$. No effect on adherence was observed when comparing the test groups. The use of cold plasma together with the addition of silver nanoparticles seems to be a promising strategy in the prevention of prosthetic stomatitis, reducing fungal adhesion in acrylic resins.

KEYWORDS: Oral Candidiasis. Stomatitis. Nanotechnology..

\section{INTRODUÇÃO}

Biofilme é uma estrutura embebida em uma matriz extracelular, sendo constituído por meio de crescimento microbiano, o qual está relacionado ao desenvolvimento e evolução de infecções fúngicas e bacterianas (UTTER; WELCH; BORISY, 2016). Este arcabouço serve de nicho ecológico aos agentes patogênicos, uma vez que microrganismos constituintes do biofilme são mais tolerantes a agentes antimicrobianos, bem como às defesas do hospedeiro (NAM, 2014). Logo, a formação do biofilme fúngico está associado a grande ocorrência de estomatite bucal em portadores de próteses, visto que nesta população a sua recorrência é evidente (ACOSTA-TORRES et al., 2012; DAGISTAN et al., 2009). 
O fungo denominado de Candida albicans é considerado como o principal agente fúngico causador deste agravo infeccioso, sendo encontrado em cerca de $60 \%$ e $100 \%$ dos usuários de próteses dentárias DAGISTAN et al., 2009; WADY et al., 2012; ANDREOLA, 2016). Apesar da C. albicans ser o agente etiológico fúngico mais comumente isolado na cavidade bucal, estudos apontam para uma grande ocorrência de infecções fúngicas provocada por espécies de Candidas não albicans, sendo, no Brasil, a Candida tropicalis a espécie mais encontrada (ACOSTA-TORRES et al., 2012; ANDREOLA, 2016).

Nesse contexto, a inclusão de dispositivos protéticos tendo como base materiais poliméricos insere um novo nicho ecológico, o qual torna-se apropriado à formação do biofilme produzido pela Candida sp. (MONTEIRO et al., 2009). Assim, o uso prolongado da prótese sem a adequada remoção e higienização é fator primordial para que a estomatite protética tenha alta prevalência (UTTER; WELCH; BORISY, 2016; NAM, 2014). Portanto, a inclusão de estratégias antimicrobianas de controle do biofilme é necessária, visto que há dificuldade, por parte do usuário da prótese, em controlá-lo (NAM, 2014; CASTRO et al. 2016). Neste cenário, a incorporação de nanopartículas de prata (AgNPs) ou uso de plasma a baixa pressão e temperatura para o controle de tal estrutura apresenta a seguintes vantagens: as AgNPs são bem toleradas pelos tecidos e exibem um perfil de baixa toxicidade (WADY et al., 2012; MONTEIRO et al., 2009; CASTRO et al. 2016); o gás de plasma é uma fonte limpa que apresenta grande quantidade de espécies reativas, tais como elétrons, íons, nêutrons excitados eletronicamente e radicais livres, modificando sua morfologia através da remoção de elétrons das moléculas (COLOMBO; MATAFORA; MORO, 2014; PORCIUNCULA et al., 2015). No entanto, ainda não há, até o presente momento, relatos de resultados que informem o potencial antimicrobiano comparativo destes 2 compostos individuais ou de modo associado, sendo necessária uma ampliação de estudos nesta área.

Considerando-se que a adição da AgNPs ou do plasma de baixa pressão e temperatura sobre a superfície de poli(metacrilato de metila) (PMNA) impediria a formação e a evolução da estomatite protética provocada pela Candida sp., bem como levando em consideração a hipótese de que a adição destes biomateriais subtrairia a necessidade da terapêutica medicamentosa, o presente estudo tem o objetivo de investigar a influência da adição de AgNPs e/ou plasma a baixa pressão e temperatura na adesão da C. albicans em superfícies de base de poli (metacrilato de metila).

\section{MATERIAIS E MÉTODOS}

Foram confeccionados, a partir de resina acrílica termopolimerizável (Trilux Base, VIPI, Pirassununga, Brasil), 80 corpos de prova nas seguintes dimensões: 2 centímetros $(\mathrm{cm}) \times$ $1 \mathrm{~cm} \times 3$ milímetros $(\mathrm{mm})$. Os mesmos foram subdivididos em 4 grupos, conforme segue exemplificado no Quadro 1. A queda pode ser classificada em:

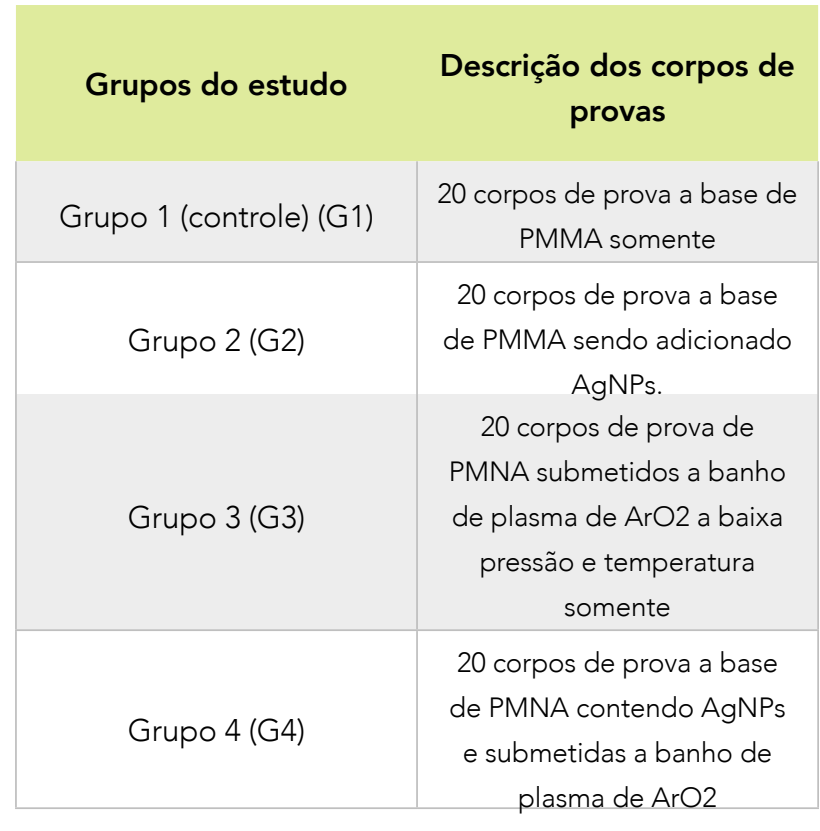

PMMA: poli(metacrilato de metila); AgNPs: nanopartículas de prata; ArO2: mistura de argônio e oxigênio. 
Síntese de Nanopartículas de prata

Para a síntese das AgNPs, seguiu-se a metodologia conforme descrita anteriormente por Lee e Meisel (1982). Tal metodologia baseia-se na redução pelo Borohidreto de sódio (NaBH4) (Labsynth Produtos para laboratório Ltda, Diadema, São Paulo) utilizando como veículo a água destilada (Asfer Indústria Química, São Caetano do Sul, São Paulo). Logo, realizou-se uma suspensão constituída por $30 \mathrm{ml}$ de $\mathrm{NaBH} 4$ a 2 milimolar (mM). A mesma foi devidamente acondicionada em um recipiente vítreo e imersa em banho de gelo a uma temperatura de $3^{\circ} \mathrm{C}$. Posteriormente, foram gotejados, a velocidade de 1 gota por segundo (seg), $10 \mathrm{ml}$ de uma solução de nitrato de prata (AgNO3) (Dinâmica Química Contemporânea Ltda, Diadema, São Paulo) a 0,001M. A solução foi mantida sob agitação mecânica (Essence Dental Importação e Exportação Ltda, Araraquara, São Paulo) durante o gotejamento, na qual foi percebida a reação química pela mudança de coloração do líquido, o qual passou de incolor para acinzentado. Após gotejamento durante 7 minutos, a solução de AgNPs foi levada a temperatura ambiente para estabilização.

Sequencialmente à realização deste procedimento e conforme estudo publicado previamente por Koruglu et al. (2016), 18 microlitros $(\mu \mathrm{L})$ da solução de AgNPs a uma concentração 0,3\% foram incorporados ao monômero. Esta escolha deve-se à observação de melhores características térmicas e mecânicas quando 0,3\% foi utilizado.

\section{Banho de Plasma à baixa pressão e temperatura}

Os corpos de prova foram acondicionados em água destilada a $37^{\circ} \mathrm{C}$ durante 48 horas. Para realização do banho de plasma foi utilizado um tubo de teflon cilíndrico de $5 \mathrm{~cm}$ de circunferência e $30 \mathrm{~cm}$ de comprimento. Com o auxílio de uma bomba a vácuo (Master Ink, Maringá, Paraná) foi gerada uma pressão interna inicial de 2 Pascal ( $\mathrm{Pa}$ ). Neste momento, o reator foi preenchido com a adição de uma mistura contendo $98 \%$ de Ar e $2 \%$ de $\mathrm{O} 2$ com fluxo de 5 Litros/minuto (L/min). Portanto, exerceu-se uma pressão interna de $10 \mathrm{~Pa}$. O plasma foi gerado no interior deste tubo de teflon, através da ação de um campo magnético a uma voltagem de 18 kilovolts (kV). Os corpos de prova foram mantidos a uma distância de 10 $\mathrm{mm}$ da saída do tubo de teflon durante um período de $90 \mathrm{seg}$. A escolha deste tempo deve-se aos resultados prévios obtidos pelo nosso grupo (dados não publicados) de que não há diferença significativa na adesão de Candida albicans quando o jato de plasma foi utilizado por 120 segundos (maior tempo considerável) e 90 segundos.

\section{Polimento}

O polimento foi realizado com o auxílio de uma politriz de bancada, seguindo a sequência de discos preconizada pelo fabricante.

\section{Preparação do inóculo para análise de aderência fúngica}

Foi utilizada uma cepa de origem clínica de Candida albicans (ATCC 90028) (Microbiologics ${ }^{\circledR}$, St. Cloud, EUA). A mesma foi reisolada em Ágar SabouraudCloranfenicol (SAB-Cl) (Difco-BD Diagnostic Systems, Le Ponte de Craix, França) e, então, reincubada em estufa microbiológica a $37{ }^{\circ} \mathrm{C}$ por 24 horas, em aerobiose. Uma suspensão de leveduras a 106 células/ $\mathrm{mL}$ foi padronizada através da contagem em câmara de Neubawer (Kasvi, Padova, Itália). Uma alíquota de $200 \mu \mathrm{L}$ desta suspensão padronizada foi, então, dispensada no interior de um tubo de falcon estéril. A este inóculo adicionou-se 1,600 $\mu \mathrm{L}$ caldo infusão cérebro coração (BHI) (Difco, Le Ponte de Craix, França) e $200 \mu \mathrm{L}$ de saliva artificial (Bellafarma, Caxias do Sul, Brasil). 


\section{Determinação da adesão fúngica}

Para análise da adesão fúngica, os corpos de prova de todos os grupos do estudo foram transferidos para tubos de Falcon (TPP Techno Plastic Products AG, Transadingen, Suíça), contendo a suspensão supracitada. Após esta transferência, estes tubos foram incubados em aerobiose por 48 horas a $37^{\circ} \mathrm{C}$. Após este período, os corpos de prova foram transferidos para novos tubos de falcon estéreis e a eles foram adicionados 2 $\mathrm{ml}$ de soro fisiológico $(\mathrm{NaCl} 0,9 \%)$, os quais foram agitados por meio de um agitador tipo Vortex (Kasvi, Padova, Itália) para remoção das células fúngicas aderidas aos corpos de prova durante 2 min. Posteriormente, $100 \mu \mathrm{L}$ da suspensão foi semeada na superfície de placas contendo SAB-Cl. As mesmas foram incubadas em aerobiose a $37^{\circ} \mathrm{C}$ por 24 horas. Posteriormente, houve o estabelecimento das Unidades Formadoras de Colônias por mililitro (UFC/mL) de forma manual.

\section{Análise estatística}

Os dados coletados foram analisados utilizando o programa GraphPad 4.0 para Windows (GraphPad Software INC., La Jolla, CA). Para análise entre todos os grupos do estudo foi utilizado ANOVA one-way com pós-teste de Tukey's. Os resultados foram considerados estatisticamente significativos quando $P \leq 0,05$. Utilizou-se um intervalo de confiança (IC) de 95\%.

\section{RESULTADOS}

No que diz respeito ao G1 (grupo-controle), observou uma média de aderência fúngica igual a 4,49 (1.70-7.9; \pm 1.83 ; IC 95\%). No G2, o valor médio de aderência foi igual a 0,26 (0.0-1.02; \pm 0.41 ; IC 95\%). No G3, foi observada uma adesão de C. albicans de 0,17 (0.05-0.5; $\pm 0.09 ;$ IC 95\%). No G4, houve uma adesão de microrganismos aderidos de 0,03 (0.0-0.2; \pm 0.05 ; IC 95\%). A tabela 1 descreve os resultados dos referidos grupos deste estudo.

\begin{tabular}{|c|c|}
\hline Grupo do estudo & $X(P z \pm d p)$ \\
\hline G1 -Resina acrílica & $4,49(1.70-7.9 \pm 1.83) a, b, c$ \\
\hline $\begin{array}{c}\text { G2 - Resina acrílica }+ \\
\text { AgNPs }\end{array}$ & $\begin{array}{c}0,26(0.0-1.02 \pm 0.41) a, b \\
d, e\end{array}$ \\
\hline $\begin{array}{c}\mathrm{G} 3 \text { - Resina acrílica }+ \\
\mathrm{ArO} 2\end{array}$ & $\begin{array}{c}0,17(0.05-0.5 \pm 0.09) b, c \\
d, f\end{array}$ \\
\hline $\begin{array}{c}\text { G4 - Resina acrílica + } \\
\text { ArO2 + AgNPs }\end{array}$ & $0,03(0.0-0.2 \pm 0.05) d, e, f$ \\
\hline \multicolumn{2}{|c|}{$\begin{array}{l}\text { X: média; PZ: amplitude do intervalo; } \pm \text { : desvio } \\
\text { padrão } P<0,0001 \mathrm{~A} \\
\text { a, b, c: significante; e, f: não significante } \\
\text { Fonte: Autoria própria (2018). }\end{array}$} \\
\hline
\end{tabular}

Tabela 1 - Aderência da C. albicans em poli(metacrilato de metila) após a adição de nanopartículas de prata e/ou

banho de plasma de baixa pressão e temperatura.

Quando o G1 foi comparado aos demais grupos do estudo, demonstrou-se uma diferença significativa na adesão fúngica $(P<0,0001)$. Porém, quando os grupos-testes foram comparados entre si (G2 x G3; G2 x G4; G3 x G4) não foi observada diferença significativa entre eles $(-0.6687$ a 0.8630 (IC $95 \%) ;-.05363$ a 0.9954 (IC 95\%). -0.6335 a 0,8982 (IC 95\%), respectivamente). A Figura 1 exemplifica as diferenças na adesão fúngica entre os grupos 1, 2, 3 e 4 .

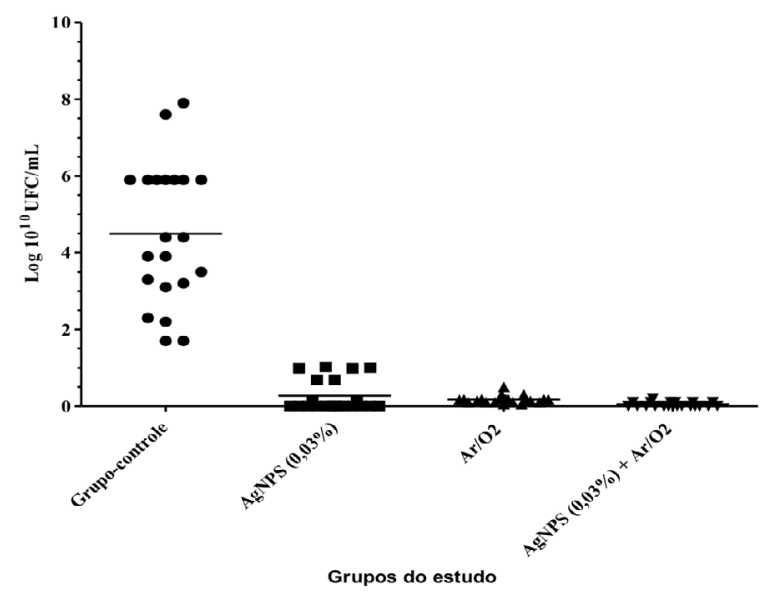

Gráfico 1 - Grau de adesão fúngica relacionado a C. albicans em superfície de poli(metacrilato de metila)

Fonte: Autoria própria (2018). 


\section{DISCUSSÃO}

Diversos estudos demonstram que dispositivos intraorais favorecem o acúmulo de biofilme (UTTER; WELCH; BORISY, 2016; NAM, 2014; ACOSTA-TORRES et al., 2012; DAGISTAN et al., 2009). Esta adesão é favorecida pelas características morfológicas e genéticas dos microrganismos em associação às características do material, tal como a hidrofobicidade (WADY et al., 2012; ANDREOLA, 2016; MONTEIRO, 2009; CASTRO et al., 2016). Neste cenário, após a disseminação celular e a estabilização do biofilme, estes micro-organismos tornam-se mais resistentes a agentes antimicrobianos (UTTER; WELCH; BORISY, 2016; COLOMBO; MATAFORA; MORO, 2014; PORCIUNCULA et al., 2015).

Sob esta perspectiva, a adesão é uma etapa primordial para a colonização e posterior infecção por Candida spp. Nas etapas subsequentes do biofilme, há a expansão e a maturação que estão dimensionadas à superfície através do aumento da secreção de matriz extracelular (EPS), bem como há dispersão que se caracteriza pelo caráter crônico e pela liberação de novas células que contribuem para o aumento da adesão, oferecendo ao biofilme uma característica cíclica (MONTEIRO et al., 2009; CASTRO et al., 2016; LEE; MEISEL, 1982). Estes fatores associados à higiene deficiente, bem como atrelados às características do material, favorecem o desenvolvimento de estomatite protética (ACOSTA-TORRES et al., 2012; KOROGLU et al., 2016; PETROVIĆ, 2018).

Atualmente, a nanotecnologia utiliza os benefícios da prata (Ag), uma vez que esta possui um amplo espectro antimicrobiano, sendo incorporada em bases protéticas (ANDREOLA, 2016; COLOMBO; MATAFORA; MORO, 2014; NAM, LEE, C. L., LEE, C. J., 2012). Wady et al. (2012) determinaram que solução de AgNPs apresenta efeito fungicida contra leveduras de C. albicans em estágio plantônico. Entretanto, neste estudo, os autores também observaram que após a utilização de AgNPs - produzidas pela metodologia utilizando álcool polivinílico (PVA) a 1000, 750, 500, 250 e 30 partes por milhão (PPM) - houve alteração da hidrofobicidade da resina sem observar diferenças significativas na formação do biofilme da C. albicans sobre a superfície de tais corpos de prova. Resultados completamente distintos foram aqui encontrados, uma vez que se observou uma diferença significativa na adesão fúngica da C. albicans quando da inclusão de 0,3\% de AgNPs obtidas pela técnica de $\mathrm{NaBH} 4$ aos corpos de prova. Provavelmente, esta diferença de resultados se deve ao método utilizado para obtenção da AgNPs, ao tamanho das nanopartículas geradas e à metodologia para quantificação do biofilme. Em contrapartida, os dados do nosso estudo corroboram com os resultados encontrados por Acosta-Torres et al. (2012) e Castro et al. (2016) que também avaliaram a adesão da C. albicans em discos de resina acrílica contendo AgNPs, bem como vanato de prata nanoparticulado (b-AgVO3), respectivamente. Estes autores também relataram que, após testes microbianos, os corpos de prova, aos quais foram adicionados AgNPs, apresentaram menor aderência de $\mathrm{C}$. albicans quando comparados a discos que continham apenas a resina comercial. Nossos resultados também dão suporte a relatos encontrados por Monteiro et al. (2009) e Nam, Lee, C. L., Lee, C. J. (2012). Dessa forma, torna-se clara a influência da adição de AgNPs, as quais agregam suas propriedades antifúngicas diretamente sobre a superfície protética.

mecanismo de ação da Ag está baseado na forte união de grupos doadores de elétrons sobre moléculas biológicas, as quais contêm enxofre $(\mathrm{S})$, oxigênio (O2) e nitrogênio (N2) (WADY et al., 2012; MONTEIRO et al., 2009). Assim, a adição de AgNPs a biomateriais altera o metabolismo microbiano, fato que impede a proliferação de micro-organismos patogênicos. Quanto ao tamanho das partículas de Ag, estudos recentes evidenciaram que partículas menores são mais eficazes 
comparadas a grandes partículas. Isto deve-se à maior área de superfície de contato gerada (NAM, 2014; KOROGLU et al., 2016; RAl; YADAV; GADE, 2009). Sendo assim, neste estudo, a síntese de AgNPs através da redução por Borohidreto de sódio ( $\mathrm{NaBH} 4)$ contribuiu para que se formassem partículas menores. Assim, tanto o tamanho quanto a morfologia das partículas atuam como fator influenciador na atividade biocida.

Quanto à ação antimicrobiana do gás plasma gerado à baixa pressão e temperatura, um estudo realizado por Sladek et al. (2004) avaliou sua ação em diferentes micro-organismos, tais como: Lactobacillus casei, Streptococcus mutans, Candida albicans e Escherichia coli. Neste estudo, os autores concluíram que há eficácia antimicrobiana na superfície abiótica com o uso do plasma, sendo $\circ$ S. mutans o micro-organismo mais resistente após a ação do plasma. Adicionalmente, os resultados obtidos com a utilização do plasma de $\mathrm{ArO} 2$, realizados por Pan et al. (2015), também demonstraram uma redução efetiva na adesão de $C$. albicans. Interessantemente, nosso estudo dá suporte aos resultados encontrados por este grupo no que diz respeito à adesão fúngica no que se refere ao uso de plasma à baixa pressão e temperatura.

Ademais, também se torna de extrema importância uma abordagem sobre dados da toxicidade gerada com o uso de Ag nanoparticulada. Tal toxicidade destas nanopartículas estão baseadas nas características de letalidade celular hepática e cerebral em modelos animais, uma vez que há facilidade do rompimento das membranas celulares devido às finas partículas. Estudos de toxicidade em algas, plantas e micro-organismos indicam que na Escherichia coli uma concentração variável de 50-60 microgramas por centímetro cúbico $(\mu \mathrm{g} / \mathrm{cm} 3)$ promove a inibição do crescimento bacteriano. Já no gênero Saccharomyces, concentrações de 13.2 nanomolar (nM) induzem a perda de permeabilidade da membrana (TRIPATHI et al., 2017). Por conseguinte, estudos de doses letais (DL) relatam que a DL50 Oral em modelos animais é de 1.173 miligramas por quilograma $(\mathrm{mg} / \mathrm{Kg})$ e que a degeneração hepática em ratos machos ocorre com a presença de $1 \mu \mathrm{g} / \mathrm{mL}$ (SIQUEIRA et al., 2013). Além disso, dados experimentais apontam que, dependendo da dose e do tempo de exposição, a prata pode provocar efeitos dermatológicos, imunológicos, efeitos neurológicos e perda de peso em humanos. No caso de exposição oral, seus efeitos sistêmicos ocorrem devido à má absorção dos compostos de prata a partir do trato intestinal. Considera-se que uma dose de 10 gramas (gr) de AgNO3 tomadas por via oral seja letal ao homem (IPCS, 1977). No entanto, não há dados na literatura sobre a morte de humanos pela exposição às nanopartículas de prata. Neste contexto, a concentração de AgNPs utilizadas na incorporação ao PMMA em nosso estudo está abaixo do índice considerado letal em humanos, uma vez que $18 \mu \mathrm{L}$ da solução de AgNPs a uma concentração 0,3\% foi incorporada ao monômero. Adicionalmente, os dados sobre a toxicidade com o uso de plasma à baixa pressão e temperatura também são limitados. Interessantemente, Liu et al. (2011) realizaram um estudo na mucosa oral de coelhos para avaliar o grau de citotoxicidade celular através de danos teciduais após o uso de plasma de $\mathrm{He} / \mathrm{O} 2$. Os autores deste estudo revelaram que não foi observada nenhuma modificação patológica nos tecidos após uso da tecnologia. Sendo assim, estes autores concluíram que plasma à baixa pressão e temperatura apresenta efeito bactericida satisfatório para a erradicação do biofilme sem ocasionar danos teciduais.

Adicionalmente, o presente estudo também comparou o efeito antimicrobiano no tocante ao uso de banho de plasma de ArO2, somente (G3) x adição de AgNPs associado a banho de plasma (G4). Os resultados obtidos com este ensaio demonstraram uma redução significativa na 
adesão de C. albicans quando estes corpos de prova foram comparados aos corpos de prova do grupo-controle (G1). No entanto, não houve diferença significativa quando os grupos-testes foram comparados entre si. Portanto, nossos resultados evidenciam uma ação efetiva na redução da adesão fúngica da C. albicans nos 2 tratamentos de superfície, sejam eles usados de maneira individual ou de modo associado. Entretanto, demais comparações não podem ser aqui realizadas devido ao fato de a literatura não apresentar estudos sobre aderência fúngica, os quais avaliam a ação de AgNPs e banho plasma de forma associada.

Por conseguinte, estudos recentes também evidenciam o uso desta tecnologia em diferentes especialidades da Odontologia (COLOMBO; MATAFORA; MORO, 2014; PORCIUNCULA et al., 2015). Dessa forma, diversas pesquisas evidenciam 0 uso promissor do plasma na Odontologia, a fim de reduzir o número de patógenos e viabilizar uma melhor descontaminação de superfícies (LIU et al., 2011; RITTS at al., 2010; HABIB; HOTTEL; HONG, 2014; PAN et al., 2010; SILVA et al., 2011). Para tanto, a influência desta tecnologia deve-se ao fato de que ela propicia interações químicas com a superfície dos materiais em bases de PMMA (poli(metacrilato de metila)), uma vez que a utilização do gás de plasma apresenta a vantagem de manter inalteradas as características do seu interior (PORCIUNCULA et al., 2015). Isto deve-se a parcial ionização dos gases, os quais são gerados em pequenas dimensões (COLOMBO; MATAFORA; MORO, 2014).

Em resumo, os estudos sobre a atividade antifúngica da prata e a sua redução na adesão de biofilme em superfícies de bases protéticas são promissores. Em adição a isso, o uso do plasma também se apresenta como metodologia adequada na Odontologia, visto a sua capacidade antimicrobiana, bem como a vantagem da não alteração dos materiais em que são empregados. Porém, são ainda inexistentes na literatura estudos da utilização do plasma em concomitância com AgNPs, sendo necessárias ampliações de estudos nesta área, uma vez que não há resultados relacionados à adesão fúngica associada a diferentes concentrações de AgNPs, bem como no estudo da formação de biofilme provocado por espécies de Candida não-albicans.

\section{CONSIDERAÇÕES FINAIS}

A partir dos resultados obtidos, a utilização de AgNPs associada ou não ao banho de plasma de baixa temperatura e pressão, torna-se uma estratégia promissora na prevenção de adesão de C. albicans em superfícies protéticas, beneficiando os usuários destas, podendo ser empregada em ambiente clínico. Todavia, são necessários mais estudos longitudinais. 


\section{REFERÊNCIAS BIBLIOGRÁFICAS}

ACOSTA-TORRES, L. S. et al. Cytocompatible antifungal acrylic resin containing silver nanoparticles for dentures. International Journal of Nanomedicine, v. 7, p. 4777-4786, 2012.

ANDREOLA, P. Comparative study between extracellular phospholipase and proteinase production in clinically important of the genus Candida isolated in oral candidiasis patients. Revista de Odontologia da UNESP, v. 45, p. 219-226, 2016.

CASTRO, D. T. de. et al. Evaluation of antibiofilm and mechanical properties of new nanocomposites based on acrylic resins and silver vanadate nanoparticles. Archives of Oral Biology, v. 67, p. 46-53, jul. 2016.

COLOMBO, L. M. P. C.; MATAFORA, F. L.; MORO, A. F. V. Condicionamento de superfícies na Odontologia com plasma de argônio: uma revisão de literatura. Revista Brasileira de Odontologia, v. 71, p. 85-88, 2014.

DAGISTAN, S. et al. Differential diagnosis of denture-induced stomatitis, Candida, and their variations in patients using complete denture: a clinical and mycological study. Mycoses, v. 52, p. 266-271, 2009.

HABIB, M.; HOTTEL, T. L.; HONG, L. Antimicrobial effects of nonthermal atmospheric plasma as a novel root canal disinfectant. Clinical Plasma Medicine, v. 2, p. 17-21, 2014.

INTERNATIONAL PROGRAMME ON CHEMICAL SAFETY - IPCS. Twenty-first Report of the Joint FAO/WHO Expert Committee on Food Additives. Geneva, 1977. Disponível em: <http://www.inchem. org/documents/jecfa/jecmono/v12je19.htm>. Acesso em: 13 out. 2018.

KOROGLU, A. et al. Silver nanoparticle incorporation effect on mechanical and thermal properties of denture base acrylic resins. Journal of Applied Oral Science, v. 24, p. 590-596, 2016.

LEE, P.; MEISEL, D. Adsorption and surface-enhanced Raman of dyes on silver and gold sols. The Journal of Physical Chemistry, v. 86, p. 3391-3395, 1982.

LIU, D. et al. Bacterial-killing effect of atmospheric pressure nonequilibrium plasma jet and oral mucosa response. Journal of Huazhong University of Science and Technology Medical Sciences, v. 31, p. 852-856, 2011. 
MONTEIRO, D. R. et al. The growing importance of materials that prevent microbial adhesion: antimicrobial effect of medical devices containing silver. International Journal of Antimicrobial Agents, v. 34, p. 103-110, 2009.

NAM, K. Y. Characterization and bacterial anti-adherent effect on modified PMMA denture acrylic resin containing platinum nanoparticles. Journal of Advanced Prosthodontics, v. 6, p. 207-214, 2014.

NAM, K.Y.; LEE, C. L.; LEE, C. J. Antifungal and physical characteristics of modified denture base acrylic incorporated with silver nanoparticles. Gerodontology, v. 29, p. 413-e419, 2012.

PAN, H. et al. Cold plasma-induced surface modification of heatpolymerized acrylic resin and prevention of early adherence of Candida albicans. Dental Materials Journal, v. 34, p. 529-536, 2015.

PAN, J. et al. A novel method of tooth whitening using cold plasma microjet driven by direct current in atmospheric pressure air. IEEE Transactions on Plasma Science, v. 38, p. 3143-3151, 2010.

PETROVIĆ, M. et al. Fungicidal PMMA-Undecylenic acid composites. International Journal of Molecular Sciences, v. 19, n. 1, p. 184-200, 2018.

PORCIUNCULA, M. et al. Tecnologia de plasma na Odontologia: revisão de literatura. Revista Brasileira de Odontologia, v. 72, p. 100103, 2015.

RAI, M.; YADAV, A.; GADE, A. Silver nanoparticles as a new generation of antimicrobials. Biotechnology Advances, v. 27, p. 76-83, 2009.

RITTS, A. C. at al. Dentin surface treatment using a non-thermal argon plasma brush for interfacial bonding improvement in composite restoration. European Journal of Oral Science, v. 118, p. 510-516, 2010.

SILVA, J. S. P. et al. Osteoblastlike cell adhesion on titanium surfaces modified by plasma nitriding. International Journal of Oral \& Maxillofacial Implants, v. 26, p. 237-244, 2011.

SIQUEIRA, M. C. et al. Caracterização e avaliação da toxicidade de nanopartículas de prata para incorporação em matriz polimérica para uso em embalagens de alimentos. Embrapa Instrumentação, v. 1, p. 542-544, 2013.

SLADEK, R. E. J.; STOFFELS, E.; WALRAVEN, R. Plasma treatment of dental cavities: a feasibility study. IEEE Transaction on plasma Science, v. 32, p. 1540-1543, 2004. 
TRIPATHI, D. K. et al. Uptake, Accumulation and Toxicity of Silver Nanoparticle in Autotrophic Plants, and Heterotrophic Microbes: A Concentric Review. Frontiers in Microbiology, v. 8, p. 07-23, 2017.

UTTER, D. R.; WELCH, J. L. M.; BORISY, G. G. Individuality, stability, and variability of the plaque microbiome. Frontiers in Microbiology, v. 7, p. 1-13, 2016.

WADY, A. F. et al. Evaluation of Candida albicans adhesion and biofilm formation on a denture base acrylic resin containing silver nanoparticles. Journal of Applied Microbiology, v. 112, n. 6, p. 116372, 2012. 